\title{
Empirical directives in defeating adolescent depression in Oman: a systematic review
}

\begin{abstract}
Introduction: Depression is one of the major problems that affect the mental health of adolescents. In the Sultanate of Oman, adolescents account for $18.69 \%$ of the population. Untreated depression among adolescents leads behavioral deviations, increased tendency towards substance abuse, and suicidal tendencies. The low trends in seeking treatment for depressive disorders in the country, prompts the need for School Based Interventions (SBI) to identify and eradicate the problem at its roots.

Methods: Electronic searches from CINAHL, Medline, Scopus, Science direct, Springer link, and Up to date to retrieve evidence based literature on school based interventions among children and adolescents with depression was conducted. The Methodological quality of the studies chosen was ranked utilizing the rating system for hierarchy of evidence: quantitative questions. A total of 9 journal articles were included in the review.

Results: Four major themes were crystallized from the systematic review: approaches to SBI, ingredients of SBI; outcomes of SBI, and future directive for SBI. The reviewed literature was itself to be incorporated within the contexts of the Beyond Blue Conceptual Framework (BBCF), which will guide future researchers to implement empirically sound school based interventions.

Conclusion: The need for schools to incorporate a well-structured comprehensive depression preventive program is the ultimate recommendation of this empirical venture. The outcomes achieved by the various fragments of interventions serves as the forecasted benefits to fight against the disastrous and disabling depression among school children
\end{abstract}

Volume 9 Issue 6 - 2018

\author{
Joshy Abraham,' Jaimy Scaria² \\ 'MSN, Muscat, Oman \\ ${ }^{2}$ Clinical Instructor, AFMS, Oman
}

Correspondence: Joshy Abraham, MSN, Muscat, Oman, Tel 009689813783I,Email joshymangelore@gmail.com

Received: November 10, 2018 | Published: December 12, 2018

\section{Background}

Depression is one of the major problems that affect the mental health of adolescents. Approximately $2-5 \%$ of adolescents meet the diagnostic criteria for major depression at a given point of time, with around $10 \%$ experiencing a depressive disorder by age $16 .{ }^{1}$ In the Sultanate of Oman, adolescents account for $18.69 \%$ of the population. ${ }^{2}$ Available studies show that the prevalence of anxiety and depressive disorders among adolescents in secondary schools was high in Oman, comparable to rates reported among cross-national adults. In Oman, the findings about major depressive disorders were equally divided between serious and moderate severity and that $57 \%$ of the bipolar categories found were serious. ${ }^{3}$ These findings are considered to be alarming for Oman. A school based survey among Omani adolescents and youth estimated the prevalence of depressive symptoms at $17 \%$, the life-time prevalence of Major Depressive Disorder (MDD) at $3 \%$, and Bipolar Mood Disorder (BMD) at $1 \%{ }^{4}$ Al-Busaidi et al. ${ }^{5}$ reported that $27.7 \%$ of University students had depression. ${ }^{5}$ In Oman, the risk factors for depression among adolescents included a history of mental illness, high score in negative health locus of control, low score in positive health locus of control, not taking breakfast, poor relationship with family members, friends and teachers and physical abuse during adolescence. ${ }^{6}$ Female gender was ascertained as a strong predictor of major depressive disorder. ${ }^{4}$ Untreated depression among adolescents leads to behavioral problems such as irritability or moodiness, fighting, defiance, skipping school, running away, and sexual experimentation, ${ }^{7}$ and a range of mental health disorders, particularly anxiety disorders, substance abuse disorders, and bipolar disorders. ${ }^{8}$ Suicide is a common consequence of depression in adolescents, and accounts for the second leading cause of death in the developed world. ${ }^{9}$ Thus the period of adolescence is the phase in which a person is comprehensively molded to face the battles of their future. This challenging phase of life can cause an individual to slide into the depressive mood, if and when their path to success is inhibited or intimidated. Uncomplicated mild depression is often treated with psychosocial intervention and Cognitive Behavioral Therapy (CBT). Moderate and severe depression requires $\mathrm{CBT}$ and fluoxetine, along with specialist child and adolescent mental health services, ${ }^{10}$ Among Arab Muslim population, the barriers to seeking treatment for mental health problems include; the stigma associated with mental health problems, underreporting of mental disorders, lack of awareness about mental health, unspecialized health care provider, traditional cultural beliefs and complementary or alternative forms of treatment. ${ }^{11}$ In Oman a study of secondary school going adolescents and youths with DSM IV disorders showed a remarkably low treatment contact for any anxiety disorder (5.2\%) and for any mood disorder category (3.2\%). ${ }^{3}$ An alternate to alleviate diminished utilization of health services is School Based Interventions (SBI). A systematic review of the articles published between 1993 and 2015, on school-based intervention and child mental health concluded that, effective SBIs are beneficial if they are implemented based on evidence based cognitive-behavioral interventions. ${ }^{12}$ SBI to reduce or prevent depression has three approaches. The 'universal approach' is offered to all students, with an aim to prevent emergence of new cases or to help children with depressive symptoms. The 'targeted approach' are directed to children at high risk for developing depression or for children with sub-clinical signs of depression. The 'clinical approach' is advocated for children with morbid levels of depression. ${ }^{13,14}$ 


\section{Methods}

Electronic searches from CINAHL, Medline, Scopus, and Science direct, Springer link, and Up to date to retrieve evidence based literature on school based interventions among children and adolescents with depression was conducted. Key words for the search included depression, children, adolescents, youth, resilience, perceived social support, and school-based intervention.

1. The inclusion criteria for the current review were that

2. Study participants were children aged 5-12 years or adolescents aged $13-19$ years,

3. The primary aim was to reduce or prevent the symptoms of depression, or to build resilience,
4. The intervention was school-based (delivered as part of the school curriculum or as an after school endorsed activity targeted at school children),

5. A primary outcome measure was depressive symptomatology or diagnosis,

6. The study used randomized controlled trial (RCT) methodology, and

7. The study was published in a peer-reviewed, English language journal. Studies that fulfilled the inclusion criteria were coded, with all relevant data extracted and recorded.

The Methodological quality of the studies chosen was ranked utilizing the rating system for hierarchy of evidence: quantitative questions (Table 1). A total of 9 journal articles were included in the review.

Table 1 Rating system for the hierarchy of evidence: quantitative questions

\begin{tabular}{ll}
\hline Level & Definition \\
\hline Level I & $\begin{array}{l}\text { Evidence from a systematic review of all relevant randomized controlled trials (RCT's), or evidence-based clinical practice } \\
\text { guidelines based on systematic reviews of RCT's }\end{array}$ \\
Level II & $\begin{array}{l}\text { Evidence obtained from at least one well-designed Randomized Controlled Trial (RCT) } \\
\text { Level III }\end{array}$ \\
Level IV & Evidence obtained from well-designed controlled trials without randomization, quasi-experimental \\
Level V & Evidence from systematic reviews of descriptive and qualitative studies \\
Level VI & Evidence from a single descriptive or qualitative study \\
Level VII & Evidence from the opinion of authorities and/or reports of expert committees \\
\hline
\end{tabular}

\section{Results}

Table 2 summarizes the studies reviewed for the current study. Among the 9 studies critically reviewed, three belonged to level I, four to level II, and two in level II category.

Approaches to SBI: Universal Preventive Programs have been instituted to an all-inclusive population of school children. ${ }^{13,15-17}$ Among this universal population, adolescents were the target in a large number of empirical ventures. ${ }^{18-20}$ Among the studies which reflected on a population of school children with higher levels of depression, the strategy employed was indicated prevention programs. ${ }^{13,19,21}$

Ingredients of SBI: The activities instituted in universal preventive programs to prevent depression included, physical activity interventions; ${ }^{15}$ cognitive behavioral group intervention $;{ }^{16}$ social and emotional learning; ${ }^{17}$ mindfulness training: ${ }^{18}$ and yoga intervention. ${ }^{22}$ Cognitive behavioral intervention was the core implementation strategy in the indicated prevention programs..$^{21}$ The benefits of the programs which have adopted cognitive-behavioral model, with an aim to reduce depression among adolescents have reported, significant enhancements in cognitive restricting, and support-seeking behaviors. ${ }^{23}$ Another successful intervention utilized in depression preventive programs among adolescents is interpersonal psychotherapy - adolescent skills training program (IPT-AST). Participants enrolled into such programs had significantly lesser depressive symptoms, and improved overall functioning..$^{24,25}$ Technological advancements through computer and mobile based self-help interventions in reducing depression among school children has been empirically recorded. ${ }^{19,20}$ Randomized control trials have dominated these empirical pursuits.
These interventions were instituted by both school teaching $\operatorname{staff}^{17}$ and mental health professionals. ${ }^{21}$

Outcomes of SBI: The consequences of school based interventions have been measured by various outcomes. The predominant measurement criteria were the estimation of depression levels. A wide range of standardized tools have been employed in testing this outcome. They include Children's Depression Inventory; ${ }^{16}$ the Short Mood and Feelings Questionnaire-Child Version; ${ }^{22}$ and Center for Epidemiological Studies Depression Scale (CES-D). ${ }^{13}$ The appropriateness of the CES-D tool in evaluating depression among adolescents, was been widely reported by Radloff ${ }^{26}$ and Robert. ${ }^{27}$ Evaluation of resilience levels have been employed in many of the school based interventions to alleviate depression. The most frequented tools used for testing resilience includes Ego-Resiliency Scale ${ }^{18}$ and resilience scale. ${ }^{28}$ The lack of resilience among adolescents can lead to maladaptation and psychopathology in adulthood. ${ }^{29}$ Furthermore, 'The Penn Resilience' program concluded that empowering adolescents with self-belief and self-efficacy, helps them to overcome setbacks in life..$^{30}$ The role of social support as a coping resource among adolescents have also been empirically well established. Many researchers have concluded that social support serves as a buffer between stressful life events and symptoms of physical and psychological imbalances. The most prominent too utilized to test this domain is the Multidimensional Scale of Perceived Social Support (MSPSS). Dahlem et al. $^{31}$ had statistically established that, high levels of perceived social support, were associated with low levels of depression and anxiety among undergraduate university students. ${ }^{31}$ The Thai version of the MSPSS had yielded negative correlations with 
the Thai Depressive inventory, when tested among medical student. ${ }^{32}$ Measures of mindfulness have also been the outcome measure in empirical ventures to eradicate depression among school children. Cognitive and Affective Mindfulness Scale ${ }^{18}$ has been consistently used to test this virtue. Researcher have also reported their outcome of school based intervention by testing the school students' anxiety levels $;{ }^{16}$ psychological well-being: ${ }^{18}$ stress responses and relations with peers, ${ }^{22}$ hopelessness levels; ${ }^{19}$ and optimistic thinking style. ${ }^{13}$ Most school based intervention studies reported reduced depression levels. ${ }^{16,19}$ However the persistence of these effects was significantly

Table 2 Essentials on the literature review of school based interventions lower in these empirical pursuits. The change in the thinking style of the students were conveyed in the reviewed studies, in terms of a positive impact on responses to stress induced rumination, intrusive thoughts, and emotional arousal; ${ }^{22}$ and increased optimistic thinking. ${ }^{20}$ On the interpersonal front, manifold benefits have been reported by the researchers, like improved social and emotional skills $;{ }^{17}$ practice of agreeableness and emotional stability; ${ }^{18}$ and the perceived ability to navigate the challenges imposed by adolescence..$^{30}$ The quantifiable outcome achieved by school based interventions, was improved academic performance. ${ }^{17}$

\begin{tabular}{cccccc}
\hline $\begin{array}{c}\text { Author } \\
\text { (Year) }\end{array}$ & Aim & $\begin{array}{c}\text { Participants } \\
\text { (Sample Size n) }\end{array}$ & Intervention & Outcome Measures & Level of
\end{tabular}

$\begin{array}{lll} & \begin{array}{l}\text { To assess the } \\ \text { impact of }\end{array} & \\ & \text { Physical } & \\ & \text { Activity (PA) } & \text { Children aged } \\ \text { Brown et } & \text { interventions } & \text { / or adolescents } \\ \text { al. }^{15} & \begin{array}{l}\text { on depression } \\ \text { in children and }\end{array} & \text { aged 12-19 years } \\ & \text { adolescents } & (\mathrm{n}=581) \\ & \text { using meta- } & \\ \text { analysis. } & \end{array}$

To reduce or prevent the

Calear et al. ${ }^{21} \quad$ symptoms of depression, or to build resilience 5-12 years or adolescents aged 13-19 years,
Interventions to promote or increase physical activity
To assess the effectiveness Challen et of an 18-hr al. ${ }^{16} \quad$ cognitive behavioral group intervention

To evaluate the impact of Social

Durlak et al. ${ }^{17}$ and Emotional Learning (SEL): A Meta-Analysis
School-based the school curriculum or as an after school endorsed activity targeted at schoo children)
Beck Depressive Inventory, Children's Depression Inventory, Profile of Mood States, Hospital Anxiety and Depression Scale, Short Mood and Multiple Affect Adjective Check List, and Reynolds Child Depression Scale

Children's Depression Inventory, Center for Epidemiological Studies Depression scale, and Reynolds Adolescent Depression Scale, Beck Depression Inventory and Hospital Anxiety Depression Scale Feelings Questionnaire,

Summary treatment effect was small but significant Subgroup analyses showed that methodological (e.g. studies with both education and PA intervention; those overweight or obese groups)

At post-intervention, UKRP

Social and emotional skills, Attitudes toward self and others, Positive social behavior, Conduct problems, Emotional distress, and Academic performance
Children's Depression Anxiety Scale, and Goodman Strengths and Difficulties Questionnaire with a higher quality score; and less than 3 months in duration) and participant characteristics (e.g. singlegender studies; those targeting contributed most to the reduction in depression.

Large proportion of the programs identified were based on cognitive behavioral therapy (CBT), and delivered by a mental health professional or graduate student over 8-12 sessions. Indicated programs, which targeted students exhibiting elevated levels of depression, were found to be the most effective. Teacher program leaders and the employment of attention control conditions were associated with fewer significant effects. students reported lower levels of depressive symptoms than control group students, but the effect was small and did not persist to 1-year or 2-year follow-ups. There was no significant impact on symptoms of anxiety or behavior at any point.

Level I

Level I

Level III

Compared to controls, SEL participants demonstrated significantly improved social and emotional skills, attitudes, behavior, and academic performance. School teaching staff successfully conducted SEL programs.

\section{through high} school students.
Universal Interventions 
To advance our understanding

$\begin{array}{ll}\text { Huppert et } & \text { of the effects } \\ \text { al. }{ }^{18} & \text { of mindfulness } \\ & \text { training in }\end{array}$
adolescents
Adolescent boys in a classroom setting $(\mathrm{N}=155)$ Stress Reduction (MBSR) course in a school context
Mindfulness Based

To assess the

feasibility, acceptability, and preliminary

Mendelson et al $^{22}$

Merry et al. ${ }^{19}$ outcomes of a school-based mindfulness and yoga intervention

To evaluate the effectiveness of SPARX (Smart, Positive, Active, Realistic $\mathrm{X}$-factor thoughts) a new computerized cognitive behavioral therapy intervention

To investigated the effectiveness of a universal intervention designed Sawyer et to reduce al. ${ }^{13}$ depressive symptoms among students commencing high school.

To develop and test the novel mobile phone Whittaker et delivery of al. ${ }^{20} \quad$ a depression prevention intervention for adolescents

\section{Four urban \\ public \\ schools were \\ randomized to an \\ intervention or \\ wait-list control \\ condition $(n=97$ \\ fourth and fifth \\ graders)}

187 adolescents aged 12-19,

seeking help

for depressive

symptoms, with no major risk of self-harm and deemed in need of treatment by their primary healthcare clinicians: 94 were allocated to SPARX and 93 to treatment as usual

Twenty-

five pairs of secondary schools matched on socioeconomic status were randomly assigned to either an intervention or a comparison group $(\mathrm{n}=5,634$ Year 8 students).

High school students in Auckland, New Zealand
Mindfulness program (yoga-based physical activity, breathing techniques, and guided mindfulness practices) during school hours four days per week for 12 weeks. Each intervention session lasted 45 min

\section{Computerized} cognitive behavioral therapy (SPARX) comprising seven a period of between four and seven weeks, versus treatment as usual comprising primarily face to face counselling delivered by trained counsellors and clinical psychologists.

The intervention extended over a 3 -year period and utilized a comprehensive classroom curriculum programme

Stress Questionnaire, Involuntary Engagement Coping Scale, Short Mood and Feelings Questionnaire-Child Version, Emotion Profile Inventory, and People in My Life

Children's depression rating scale, Reynolds adolescent depression scale, Mood and feelings questionnaire, Kazdin hopelessness scale for children, Spence children's anxiety scale, Paediatric quality of life enjoyment and satisfaction questionnaire, and overall satisfaction with treatment ratings

Depressive symptoms, risk and protective factors relevant to depression, and the quality of the school environment.

The intervention was developed from $15 \mathrm{key}$ messages derived from cognitive behavioral therapy (CBT).

The program was fully automated and delivered in 2 mobile phone messages/day mixture of text, video, and cartoon messages and a mobile website.

Clinician-assessed (blinded) depression symptom scores, selfrated RADS-2 depression symptom scale, incidence of depression; general and school functioning; and quality of life. modules delivered over for 9 weeks, with a
Cognitive and Affective Mindfulness Scale Revised, Ego-Resiliency Scale,

Warwick-Edinburgh

Mental Well-being scale, and Big-Five personality dimensions

Within the mindfulness group, there was a significant positive association between the amount of individual practice outside the classroom and improvement in psychologica well-being and mindfulness. Improvement in well-being was related to personality variables (agreeableness and emotional stability). Most students reported enjoying and benefiting from the mindfulness training, and $74 \%$ said they would like to continue with it in the future.

Findings suggest the intervention was attractive to students, teachers, and school administrators and that it had a positive impact on problematic responses to stress including rumination, intrusive thoughts, and emotional arousal.

Mean reduction of depression in the SPARX group. Remission rates were significantly higher in the SPARX arm than in the treatment as usual arm

Level II

Changes in the level of depressive symptoms and in the levels of risk and protective factors experienced by students in the two groups did not differ significantly over the 3 years of the study. Furthermore, statistically significant differences in the ratings of school climate across this time period were found only for staff-rated assessments.
Intervention group participants said the intervention helped them to be more positive and to get rid of negative thoughts significantly higher than proportions in the control group. 
Future directives for SBI: The reviewed articles have recommended further empirical ventures with higher methodical values, focusing on high risk population like obese adolescents; ${ }^{15}$ incorporating attention control and long-term follow-ups; ${ }^{21}$ and employing rigorous subjective and objective outcome measures. ${ }^{18}$ The studies with self-help techno-friendly interventions have recommended these interventions to address the unmet needs of the primary treatment program. ${ }^{19}$ The decrease in sustained effects of school based interventions to reduce depression, have been addressed by insisting on the need to incorporate these interventions in the school curriculum and to provide efficient training of school teachers to continue these empirically successful ventures. ${ }^{13}$

\section{Discussion}

The reviewed literature lends itself to be incorporated within the contexts of the Beyond Blue Conceptual Framework (BBCF), which will guide future researchers to implement empirically sound school based interventions. The BBCF takes its root from attachment, stress-coping and social support theories. The BBCF is based on the premise of successful psychosocial adaptation, measured in terms of emotional well-being and learning outcomes. The framework also has its stronghold on the fact that adolescence is a developmental phase with complex changes in roles, responsibilities, and relationships. The BBCF has two cardinal elements. Individual protective factors empowers an individual with vital adaptive skills and positive thinking styles. Environmental protective factors, grooms an adolescent with security and safety; social support and positive social relationships; and social connectedness and participation. Literature strongly recommends, the utilization of Cognitive Behavioral (CB) program to empower the adolescents with individual protective factors; and Interpersonal Psychotherapy - Adolescents Skills Training program (IPT-AST) ${ }^{33}$ to embed the environmental factors. CB programs focuses on unraveling cognitive distortions, cognitive restructuring strategies and self-management skills (communication, problem solving, anger management, and positive coping). ${ }^{34}$ The focus of the IPT-AST intervention encompasses three major themes of interpersonal role transitions, interpersonal role disputes, and interpersonal deficits. The consistent and constant provision of the individual and environmental protective factors will initiate the dynamic process of 'resilience' in the adolescents, which will modify the effects of adverse stressful life experiences, quantitatively demonstrated in terms of decreased levels of depression, improved resilience, and perceived social support (Figure 1). This review recommends the need for the concentrated strength of universal interventions to be followed by an on-going maintenance therapy. The focus of these on-going therapies should incorporate assessment of current levels of depression and resilience; and teacher mediated self-activating interventions. The computer and mobile based self-help interventions would suit this purpose. ${ }^{13,14}$ Training teachers ${ }^{7}$ to support these on-going processes will facilitate earlier detection of relapse and sustained effects of therapy. Furthermore, teacher can play a vital role in modifying the teachinglearning environment to foster mental health.

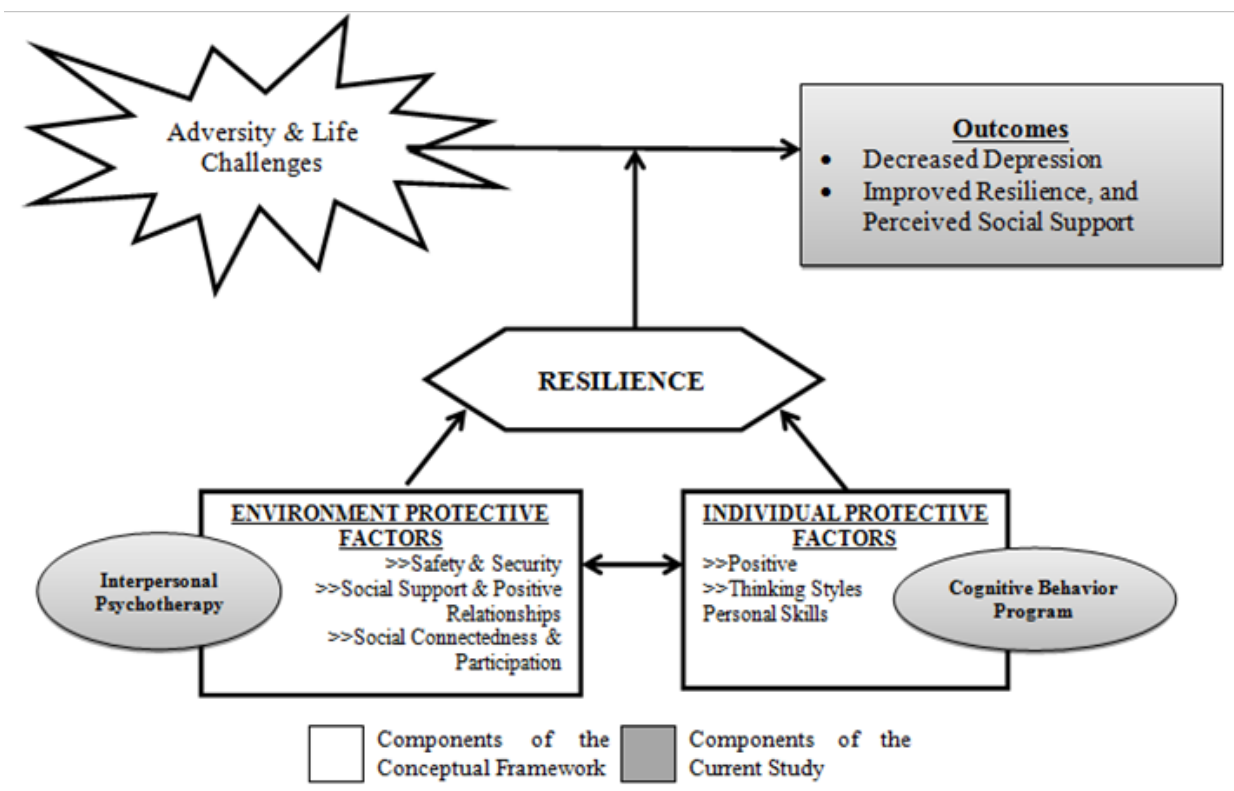

Figure I Conceptual framework based on the 'Beyond blue conceptual framework' (2005).

\section{Conclusion}

Depression among school children is endemic and the awareness of its cause and preventive strategies are prevalent. This article brings to light a systematic analysis of successfully implemented school based interventions in combating this challenge, which can adversely incapacitate school aged children. The need for schools to incorporate a well-structured comprehensive depression preventive program is the ultimate recommendation of this empirical venture. The outcomes achieved by the various fragments of interventions serves as the forecasted benefits to fight against the disastrous and disabling depression among school children.

\section{Acknowledgements}

None.

\section{Conflicts of interest}

The author declares that there is no conflict of interest.

\section{References}

1. Costello EJ, Mustillo S, Erkanli A, et al. Prevalence and development of psychiatric disorders in childhood and adolescence. Arch Gen Psychiatry. 2003;60(8):837-844. 
2. Factbook CW. Oman Demographics Profile. 2018

3. Al Riyami AA, Al Adawi SH, Al Kharusi HA, et al. Health services utilization by school going Omani adolescents and youths with DSM IV mental disorders and barriers to service use. Int J Ment Health Syst. 2009;3(1):22.

4. Jaju S, Al-Adawi S, Al-Kharusi H, Morsi M, Al-Riyami A. Prevalence and age-of-onset distributions of DSM IV mental disorders and their severity among school going Omani adolescents and youths: WMHCIDI findings. Child Adolesc Psychiatry Ment Health. 2009;3(1):29.

5. Al-Busaidi Z, Bhargava K, Al-Ismaily A, et al. Prevalence of depressive symptoms among university students in Oman. Oman Med J. 2011;26(4):235-239.

6. Afifi M, Al Riyami A, Morsi M, et al. Depressive symptoms among high school adolescents in Oman. 2006

7. Faris S. Teenage Depression: Statistics, Treatments, Symptoms, \& Diagnosis. 2012.

8. Copeland WE, Shanahan L, Costello E, et al. Childhood and adolescent psychiatric disorders as predictors of young adult disorders. Arch Gen Psychiatry. 2009;66(7):764-772.

9. Wilkinson P, Kelvin R, Roberts C, et al. Clinical and psychosocial predictors of suicide attempts and nonsuicidal self-injury in the Adolescent Depression Antidepressants and Psychotherapy Trial (ADAPT). Am J Psychiatry. 2011;168(5):495-501.

10. Thapar A, Collishaw S, Potter R, et al. Managing and preventing depression in adolescents. BMJ. 2010;340(c209):254-258.

11. Aloud N, Rathur A. Factors affecting attitudes toward seeking and using formal mental health and psychological services among Arab Muslim populations. Journal of Muslim Mental Health. 2009;4(2):79-103.

12. Paulus FW, Ohmann S, Popow C. Practitioner Review: School based interventions in child mental health. Journal of Child Psychology and Psychiatry. 2016;57(12):1337-1359.

13. Sawyer MG, Pfeiffer S, Spence SH, et al. School based prevention of depression: a randomised controlled study of the beyondblue schools research initiative. J Child Psychol Psychiatry. 2010;51(2):199-209.

14. Spence S, Burns J, Boucher S, et al. The beyondblue schools research initiative: Conceptual framework and intervention. Australasian Psychiatry. 2005;13(2):159-164.

15. Brown HE, Pearson N, Braithwaite RE, et al Physical activity interventions and depression in children and adolescents. Sports Med. 2013;43(3):195-206.

16. Challen AR, Machin SJ, Gillham JE. The UK Resilience Programme: A school-based universal nonrandomized pragmatic controlled trial. $J$ Consult Clin Psychol. 2014;82(1):75-89.

17. Durlak JA, Weissberg RP, Dymnicki AB, et al. The impact of enhancing students' social and emotional learning: A meta analysis of school based universal interventions. Child Dev. 2011;82(1):405-432.

18. Huppert FA, Johnson DM. A controlled trial of mindfulness training in schools: The importance of practice for an impact on well-being. The Journal of Positive Psychology. 2010;5(4):264-274.

19. Merry SN, Stasiak K, Shepherd M, et al. The effectiveness of SPARX, a computerised self help intervention for adolescents seeking help for depression: randomised controlled non-inferiority trial. $B M J$. 2012;344:e2598

20. Whittaker R, Merry S, Stasiak K, et al. MEMO - a mobile phone depression prevention intervention for adolescents: development process and postprogram findings on acceptability from a randomized controlled trial. J Med Internet Res. 2012;14(1):e13.

21. Calear AL, Christensen H. Review of internet-based prevention and treatment programs for anxiety and depression in children and adolescents. Med J Aust. 2010;192(11):S12.

22. Mendelson T, Greenberg MT, Dariotis JK, et al. Feasibility and preliminary outcomes of a school-based mindfulness intervention for urban youth. J Abnorm Child Psychol. 2010;38(7):985-994.

23. Wong PW, Fu K-W, Chan KY, et al. Effectiveness of a universal schoolbased programme for preventing depression in Chinese adolescents: a quasi-experimental pilot study. Journal of affective disorders. 2012;142(1-3):106-614

24. Young JF, Mufson L, Davies M. Efficacy of interpersonal psychotherapy adolescent skills training: An indicated preventive intervention for depression. Journal of Child Psychology and Psychiatry. 2006;47(12):1254-1262

25. Young JF, Mufson L, Gallop R. Preventing depression: A randomized trial of interpersonal psychotherapy adolescent skills training. Depress Anxiety. 2010;27(5):426-333.

26. Radloff LS. The CES-D scale: A self-report depression scale for research in the general population. Applied psychological measurement 1977;1(3):385-401.

27. Roberts RE, Andrews JA, Lewinsohn PM, et al. Assessment of depression in adolescents using the Center for Epidemiologic Studies Depression Scale. Psychological Assessment: A Journal of Consulting and Clinical Psychology. 1990;2(2):122-128.

28. Mota CP, Costa M, Matos PM. Resilience and deviant behavior among institutionalized adolescents: The relationship with significant adults Child and adolescent social work journal. 2016;33(4):313-325.

29. Banerjee R, Dasgupta A, Burman J, et al. Resilience level among adolescent children: a school-based study in Kolkata, India. International Journal of Contemporary Pediatrics. 2018;5(4):1641-5.

30. Reivich K, Gillham JE, Chaplin TM, et al. From helplessness to optimism: The role of resilience in treating and preventing depression in youth. Handbook of resilience in children: Springer;2013:201-214.

31. Dahlem NW, Zimet GD, Walker RR. The multidimensional scale of perceived social support: a confirmation study. Journal of clinical psychology. 1991;47(6):756-761.

32. Wongpakaran T, Wongpakaran N, Ruktrakul R. Reliability and validity of the multidimensional scale of perceived social support (MSPSS): Thai version. Clin Pract Epidemiol Ment Health. 2011;7:161-166.

33. Horowitz JL, Garber J, Ciesla JA, Young JF, Mufson L. Prevention of depressive symptoms in adolescents: A randomized trial of cognitivebehavioral and interpersonal prevention programs. $J$ Consult Clin Psychol. 2007;75(5):693-706.

34. Ackerman C. 25 CBT Techniques and Worksheets for Cognitive Behavioral Therapy. 2017 